\title{
Triggering the activation of Activin A type II receptor in human adipose stem cells towards tenogenic commitment using mechanomagnetic stimulation
}

\author{
A.I. Gonçalves, $\mathrm{PhD}^{\mathrm{a}, \mathrm{b}}, \mathrm{M}$. Rotherham, $\mathrm{PhD}^{\mathrm{c}}$, H. Markides, $\mathrm{PhD}^{\mathrm{c}}$, M.T. Rodrigues, $\mathrm{PhD}^{\mathrm{a}, \mathrm{b}}$, \\ R.L. Reis, $\mathrm{PhD}^{\mathrm{a}, \mathrm{b}, \mathrm{d}}$, M.E. Gomes, $\mathrm{PhD}^{\mathrm{a}, \mathrm{b}, \mathrm{d}, *}$, A.J. El Haj, $\mathrm{PhD}^{\mathrm{c}}$ \\ a3B's Research Group - Biomaterials, Biodegradables and Biomimetics, University of Minho, Headquarters of the European Institute of Excellence on Tissue \\ Engineering and Regenerative Medicine, Avepark - Zona Industrial da Gandra, Barco, Guimarães, Portugal \\ ${ }^{\mathrm{b}}$ ICVS/3B's - PT Government Associate Laboratory, Braga/Guimarães, Portugal \\ ${ }^{\mathrm{c}}$ Institute of Science and Technology in Medicine, Keele University, Stoke-on-Trent, UK \\ ${ }^{\mathrm{d}}$ The Discoveries Centre for Regenerative and Precision Medicine, Headquarters at University of Minho, Avepark, Barco, Guimarães, Portugal
}

Received 27 October 2017; accepted 10 February 2018

\begin{abstract}
Stem cell therapies hold potential to stimulate tendon regeneration and homeostasis, which is maintained in response to the native mechanical environment. Activins are members of the mechano-responsive TGF- $\beta$ superfamily that participates in the regulation of several downstream biological processes. Mechanosensitive membrane receptors such as activin can be activated in different types of stem cells via magnetic nanoparticles (MNPs) through remote magnetic actuation resulting in cell differentiation. In this work, we target the Activin receptor type IIA (ActRIIA) in human adipose stem cells (hASCs), using anti-ActRIIA functionalized MNPs, externally activated through a oscillating magnetic bioreactor. Upon activation, the phosphorylation of Smad2/3 is induced allowing translocation of the complex to the nucleus, regulating tenogenic transcriptional responses. Our study demonstrates the potential remote activation of MNPs tagged hASCs to trigger the Activin receptor leading to tenogenic differentiation. These results may provide insights toward tendon regeneration therapies. (C) 2018 Elsevier Inc. All rights reserved.
\end{abstract}

Key words: Human adipose derived stem cells; Magnetic nanoparticles; Activin A receptor; TGF- $\beta /$ Smad2/3 signaling pathway; Tendon tissue engineering

During tendon development, mechanical forces are transmitted to tendon cells through mechanosensitive receptors available in cell membranes. ${ }^{1,2}$ These changes initiate intracellular transduction cascades through the activation and the stimulation of transmembrane receptors such as serine/threonine kinase receptors, inducing structural changes in the cytoskeleton and promoting regulated transcriptional responses. However, the mechano-sensing mechanisms that regulate homeostasis and that

Part of the content of this manuscript was previously presented in a Poster communication at the TERMIS European Chapter Meeting 2017 in Davos, Switzerland, and in an Oral communication at the European Orthopaedic Research Society (EORS) 25th Annual \& Anniversary Meeting 2017 in Munich, Germany.

Funding: A.I.G. acknowledge the financial support from FCT/MCTES (Fundação para a Ciência e a Tecnologia/Ministério da Ciência, Tecnologia, e Ensino Superior) and the Fundo Social Europeu através do Programa Operacional do Capital Humano (FSE/POCH), PD/59/2013, for the scholarship PD/BD/113802/2015.

Authors would like also to acknowledge the EU-ITN network Mag(net)icFun for financial support.

The authors acknowledge the financial support from the European Union Framework Programme for Research and Innovation HORIZON 2020, under the TEAMING Grant agreement No 739572 - The Discoveries CTR. This article is also a result of the project (Accelerating tissue engineering and personalized medicine discoveries by the integration of key enabling nanotechnologies, marine-derived biomaterials and stem cells), supported by Norte Portugal Regional Operational Programme (NORTE 2020), under the PORTUGAL 2020 Partnership Agreement, through the European Regional Development Fund (ERDF).

Conflict of interest: A.J. El Haj is a co-founder and scientific consultant for MICA Biosystems with a $50 \%$ shareholding.

*Corresponding author at: 3B's Research Group - Biomaterials, Biodegradables and Biomimetics, University of Minho, Headquarters of the European Institute of Excellence on Tissue Engineering and Regenerative Medicine, Barco, Guimarães, Portugal.

E-mail address: megomes@dep.uminho.pt (M.E. Gomes). 


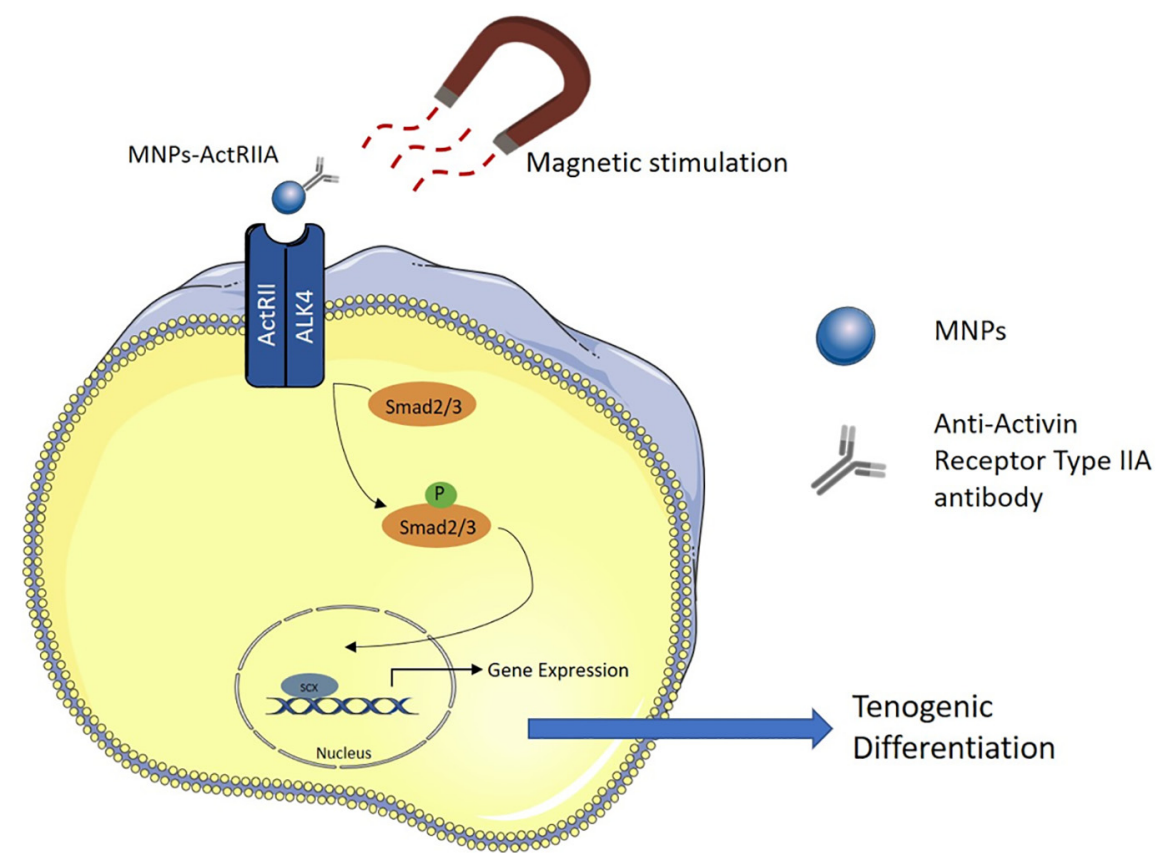

Figure 1. Schematic representation of cellular Activin A receptor targeting with MNPs functionalized with Anti-Activin type IIA antibody (MNPs-ActRIIA) for tenogenic differentiation.

are involved in mature tendon repair are not well established, hampering the development of successful cell based therapies toward tendon regeneration.

Signaling cascades are the main routes of communication between the membrane and intracellular regulatory targets. Among them, TGF- $\beta / \operatorname{Smad} 2 / 3$ was reported as one of the most relevant pathway involved in tenogenic differentiation. ${ }^{3,4}$ The transforming growth factor- $\beta$ (TGF- $\beta$ ) superfamily comprises of the TGF $\beta$, activins, NODAL, bone morphogenetic proteins (BMPs), growth and differentiation factors (GDFs) and anti-Müllerian hormone (AMH). ${ }^{5}$ Both TGF- $\beta$ and mechanical stimulation were found to activate the Smad 2 and Smad 3 molecules, downstream mediators of TGF- $\beta$ type I (activin receptor-like kinases, ALKs) and type II receptors, suggesting stress-activated TGF- $\beta$ signaling drives tenogenesis. ${ }^{6,7}$ Activin $\beta A$ has been suggested to activate SMADs pathway $^{8,9}$ and to be involved in the regulatory pathway of tenomodulin, ${ }^{10}$ a type II transmembrane glycoprotein suggested as tendon specific marker. ${ }^{11,12}$

Since tendons are notably sensitive to mechanical forces, the use of magnetic mechano-activation might constitute an effective approach to commit stem cells towards the tenogenic lineage through mechanosensing signaling cascades such as TGF- $\beta$ / Smad2/3 pathway. The technology of remote activation of mechanotransduction via magnetic nanoparticles (MNPs) has been successfully employed using the MICA Bioreactor (MICA Biosystems Ltd) to deliver the magnetic field to MNPs-tagged cells in both 2D and 3D environments. ${ }^{13,14}$ MNPs-tagged cells renders magnetic responsiveness to the engineered systems with potential to be remotely controlled and tuned by the actuation of an external magnetic field stimulating cells in vitro and upon implantation. ${ }^{15}$ Additionally, the magnetic field was shown to impact biological processes ${ }^{16,17}$ and to render a positive outcome in tissue healing. ${ }^{18,19}$ Thus, in this study we propose to investigate a magnetically actuated TE approach using an externally applied oscillating magnetic field over stem cells tenogenic phenotype commitment (Figure 1). Human adipose-derived stem cells (hASCs) were labelled with MNPs previously functionalized with anti-Activin receptor type IIA antibody. The magnetic actuation is expected to directly activate the mechanosensitive membrane receptor via functionalized MNPs, stimulating hASCs towards controlled cellular responses, more specifically regulating the transcription of tenogenic associated genes and driving differentiation. In this way, we can compare specific MNPs tagging with nonspecific effects of an oscilating magnetic field at the cell level.

Previous works using biofunctionalized MNPs targeted to the mechano-responsive ion channel TREK1, Wnt Frizzled or PDGFR $\alpha$ and $\beta$ receptors, have shown the promotion of an osteogenic, or smooth muscle cell phenotype, respectively, in hMSCs in vitro and in vivo demonstrating the potential for translation as an enabling approach for cell therapies. ${ }^{13,14,20,21}$ This raised the hypothesis that this approach can be extended to other tissues of the musculoskeletal system, such as tendons, where stem cell therapies have already been shown to be effective as veterinary treatments and would benefit from further control of differentiation. ${ }^{22}$ In this study, we have identified the cell surface receptors which are likely to be mechano-magnetically targeted and investigated if we can activate cell signalling pathways downstream.

\section{Methods}

\section{Magnetic nanoparticles (MNPs) conjugation with antibody}

Carboxyl functionalized magnetic nanoparticles (nano$\operatorname{mag}^{\circledR}$-D, 09-02-252, Micromod) were covalently coated with Anti-Activin Receptor type IIA (ActRIIA) antibody (ab135634) 
Table 1

Primers used for quantitative RT-PCR analysis.

\begin{tabular}{|c|c|c|}
\hline Gene & Sequence & NCBI reference \\
\hline \multirow[t]{2}{*}{ Human Glyceraldehyde-3-phosphate dehydrogenase $(G A P D H)$} & F- GGGAGCCAAAAGGGTCATCA & \multirow[t]{2}{*}{ NM_001256799.1 } \\
\hline & R- GCATGGACTGTGGTCATGAGT & \\
\hline \multirow[t]{2}{*}{ Collagen, Type I, alpha 1 (COL1A1) } & F- CCCCAGCCACAAAGAGTCTAC & \multirow[t]{2}{*}{ NM_000088.3 } \\
\hline & R- TTGGTGGGATGTCTTCGTCT & \\
\hline \multirow[t]{2}{*}{ Collagen, Type III, alpha 1 (COL3A1) } & F- CCTGAAGCTGATGGGGTCAA & \multirow[t]{2}{*}{ NM_000090.3 } \\
\hline & R- CAGTGTGTTTCGTGCAACCAT & \\
\hline \multirow[t]{2}{*}{ Tenascin $\mathrm{C}(T N C)$} & F- ACTGCCAAGTTCACAACAGACC & \multirow[t]{2}{*}{ NM_002160.3 } \\
\hline & R- CCCACAATGACTTCCTTGACTG & \\
\hline \multirow[t]{2}{*}{ Scleraxis $(S C X A)$} & F- AGAACACCCAGCCCAAACAGAT & \multirow[t]{2}{*}{ NM_001080514.2 } \\
\hline & R- TCGCGGTCCTTGCTCAACTTT & \\
\hline \multirow[t]{2}{*}{ Decorin $(D C N)$} & F- CTAGTCACAGAGCAGCACCTAC & \multirow[t]{2}{*}{ NM_001920.4 } \\
\hline & R- CCAGGGAACCTTTTAATCCGGGAA & \\
\hline \multirow[t]{2}{*}{ Tenomodulin (TNMD) } & F- CCGCGTCTGTGAACCTTTAC & \multirow[t]{2}{*}{ NM_022144.2 } \\
\hline & R- CACCCACCAGTTACAAGGCA & \\
\hline
\end{tabular}

herein termed as "MNPs-ActRIIA", or with Anti-Rabbit-IgG Fc antibody (ab97196), "MNPs-IgG", by carbodiimide activation as described previously. ${ }^{14}$ Briefly, particles were activated using EDAC (03449, Sigma) and NHS (130672, Sigma) dissolved in $0.5 \mathrm{M}$ MES buffer pH6.3 (Sigma) for $1 \mathrm{~h}$ at room temperature under continuous mixing. The particle suspension was washed and re-suspended in $0.1 \mathrm{M}$ MES buffer containing $60 \mu \mathrm{g}$ of anti-rabbit secondary antibody (ab97196). The particle suspension was continuously mixed overnight at $4{ }^{\circ} \mathrm{C}$ and then washed and re-suspended in $0.1 \mathrm{~mL}$ MES buffer containing $10 \mu \mathrm{g}$ of Anti-Activin Receptor type IIA antibody. Particle suspensions were mixed for $3 \mathrm{~h}$ at room temperature and then blocked with $25 \mathrm{mM}$ Glycine (Sigma) for 30 minutes before final washing and re-suspension in distilled water. Functionalized nanoparticles were then analyzed for surface charge and size using a Zetasizer $3000 \mathrm{HSa}$ (Malvern Instruments). Particles were diluted in $\mathrm{dH}_{2} \mathrm{O}$ and measurements performed at $25^{\circ} \mathrm{C}$. The size and surface charge of ActRIIA-coated nanoparticles was compared to IgG-coated nanoparticles.

\section{Cell isolation and expansion}

Human adipose-derived stem cells (hASCs) were obtained from lipoaspirate samples from the abdominal region and isolated from surplus tissue samples, under protocols previously established with Hospital da Prelada (Porto, Portugal) and with informed consent of the patients. The content of the written informed consent and related procedures were reviewed and approved by the Hospital Ethics Committee.

Human ASCs were isolated from tissue samples and cultured as described before, ${ }^{23,24}$ and have been previously characterized by RT-PCR for CD44, STRO-1, CD105 and CD90 markers. ${ }^{23}$ Briefly, the tissue was rinsed in phosphate buffered saline (PBS, Sigma-Aldrich) containing $10 \%$ of an A/A solution. The fat solution was immersed in a $0.05 \%$ collagenase type II (Sigma/ C6885) solution for $45 \mathrm{~min}$ at $37{ }^{\circ} \mathrm{C}$ under mild agitation. The digested tissue was centrifuged at $800 \mathrm{~g}$ for $10 \mathrm{~min}$ at $4{ }^{\circ} \mathrm{C}$, after which the supernatant was eliminated. Cells were expanded in basal medium composed of $\alpha$ MEM (BE12-169F, Lonza) supplemented with 10\% FBS (10270, Gibco) and 1\% A/A solution (DE17-603E, Lonza).

\section{Phosphorylation assays}

hASCs were seeded at a density of 100,000 cells/well in tissue-culture 6-well plates (Falcon) and incubated with carboxyl functionalized MNPs previously coated with anti-ActRIIA antibody or with anti-Rabbit-IgG antibody, at $25 \mu \mathrm{g}$ MNPs/ $2 \times 10^{5}$ cells. After a 30 min labelling period, in basal serum-free medium, with conjugated MNPs, hASCs were incubated/ stimulated for 2, 10, or $30 \mathrm{~min}$ in basal $\alpha$ MEM medium under magnetic stimulated conditions (magnetic field of $\geq 25 \mathrm{mT}$ from an array of permanent magnets $(\mathrm{NdFeB})$ situated beneath the culture plates at a frequency of $1 \mathrm{~Hz}$ ) with a vertical oscillating magnetic bioreactor (MICA Biosystems Ltd) and non-stimulated cells kept in identical conditions in incubator. hASCs cultured without MNPs in $\alpha$ MEM medium supplemented with Recombinant Human/Mouse/Rat Activin A Protein (338-AC-010, R\&D systems) or TGF- $\beta 3$ at 10 and $20 \mathrm{ng} / \mathrm{mL}^{25}$ were considered positive controls of ActRIIA activation. The dose of ligands was chosen based on literature and according to the phospho-Smad2/ Smad3 ELISA kit. The MNPs conjugated only with secondary IgG antibody constitute the negative control of the ActIIRA activation. After each incubation period, cells were analyzed by enzyme-linked immunosorbent assay (ELISA) that recognizes endogenous levels of phospho-Smad2 (Ser465/467) and phospho-Smad3 (Ser423/425) proteins (PathScan ${ }^{\circledR}$ Phospho-Smad2 (Ser465/467)/Smad3 (Ser423/425) Sandwich ELISA, 12001, Cell Signaling Technology), following the manufacturer's instructions.

\section{hASCs culture}

hASCs were seeded at 10,000 cells/well in 24-well plates and cultured in the following conditions for up to 14 days: i) $\alpha M E M$ medium in static conditions ("ST"), ii) $\alpha$ MEM medium in magnetically stimulated conditions ("MICA"), iii) $\alpha$ MEM medium supplemented with Activin A $(20 \mathrm{ng} / \mathrm{mL})$ in ST, iv) aMEM medium supplemented with Activin A $(20 \mathrm{ng} / \mathrm{mL})$ in 

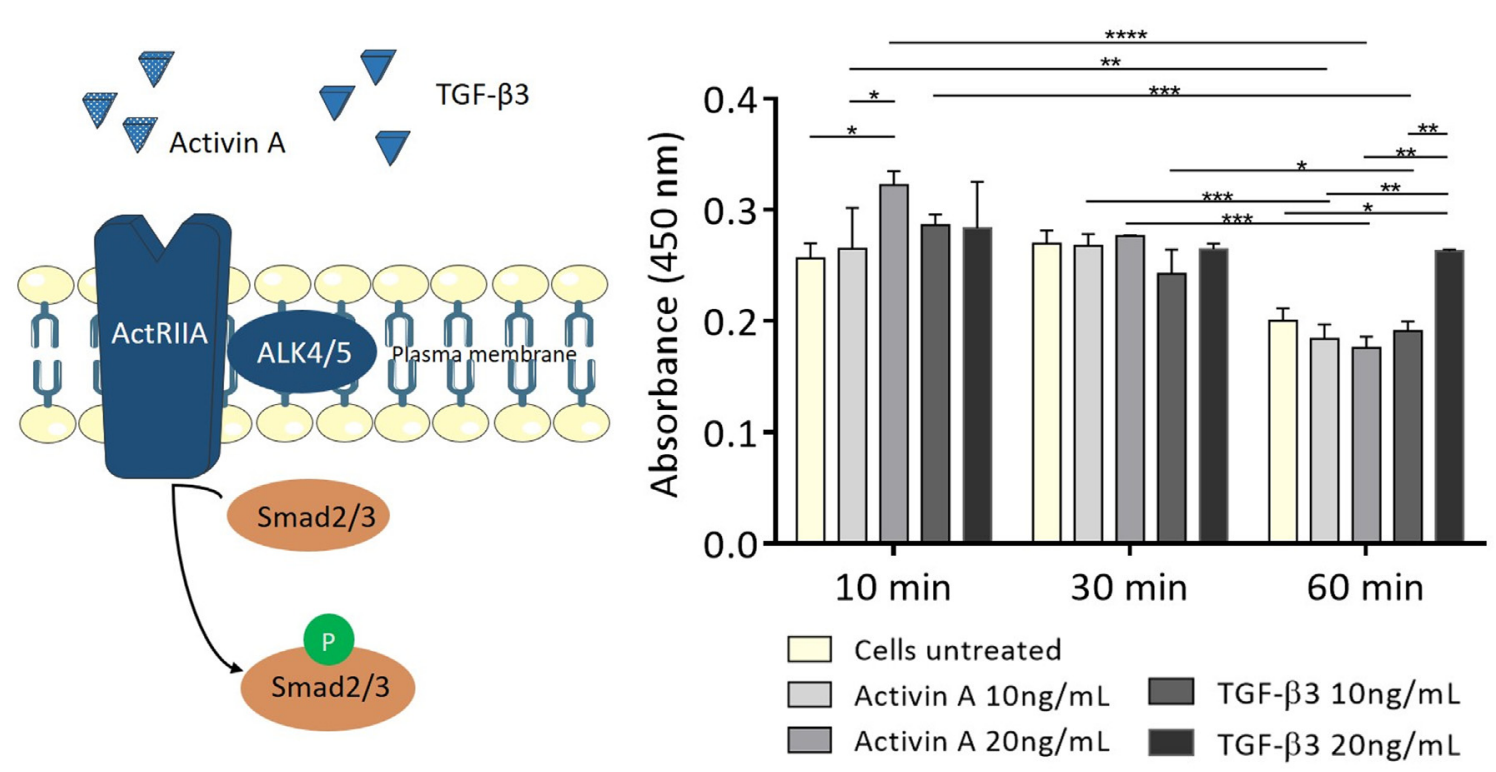

Figure 2. Phosphorylation assessment of Smad2/Smad3 in hASCs triggered by TGF- $\beta$-like ligands. (A) Schematic of the experimental design for the phosphorylation assay. Treatment of hASCs with Activin A and TGF- $\beta 3$ ligands for up to $60 \mathrm{~min}$ and subsequent phospho-Smad2/Smad3 (pSmad2/3) detection. (B) Assessment of pSmad2/3 by ELISA kit assay, in hASCs cultured in $\alpha$ MEM medium (cells untreated) or cultured in $\alpha$ MEM medium suplemented with Activin A or TGF- $\beta 3$ at 10 and $20 \mathrm{ng} / \mathrm{mL}$ for 10,30 and $60 \mathrm{~min}$. Values represent the mean $\pm \mathrm{SD}$. Symbol *, **, *** and **** denotes study groups with statistical significant difference $P<0.05, P<0.01, P<0.001$ and $P<0.0001$, respectively.

MICA, v) $\alpha$ MEM medium in the presence of MNPs-ActRIIA in ST, or vi) $\alpha$ MEM medium in the presence of MNPs-ActRIIA in MICA. Medium was changed every 3 days. Tenogenic commitment of hASCs was evaluated based on the deposition of tendon extracellular matrix (ECM) related proteins and on real time RT-PCR analysis for tendon-related markers as described in detail below.

Magnetically stimulated groups were placed in a commercially available vertical oscillating magnetic bioreactor (MICA Biosystems Ltd), providing a magnetic field of $\geq 25 \mathrm{mT}$ from an array of permanent magnets $(\mathrm{NdFeB})$ situated beneath the culture plates at a frequency of $1 \mathrm{~Hz}$, provided every other day for $1 \mathrm{~h}$ sessions. Non-stimulated groups were kept in identical conditions but without magnetic field.

\section{Dextran immunolabelling}

Fixed cells labeled with MNPs-ActRIIA or MNPs-IgG undergoing dextran immunolabelling were permeabilized with $0.025 \%$ Triton-X100 (Sigma/X100)/PBS solution, blocked with 2\% BSA (A9418, Sigma) in PBS (21-040-CVR, Corning) for $1 \mathrm{~h}$ at room temperature and incubated overnight with mouse monoclonal anti-Dextran antibody (60026FI.1, Stem Cell technologies) in $0.1 \% \mathrm{BSA}$ in PBS at $4{ }^{\circ} \mathrm{C}$. Cells were rinsed in PBS, following incubation for $1 \mathrm{~h}$ at room temperature with anti-mouse IgG FITC antibody (F2012, Sigma). Samples were stained with 4,6-Diamidino-2-phenyindole dilactate (DAPI, 40009, VWR) for 10 min and Phalloidin Tetramethylrhodamine $\mathrm{B}$ isothiocyanate (P1951, Sigma) for $20 \mathrm{~min}$. The images were acquired under a Fluorescence Inverted Microscope (Axio Observer, Zeiss).

\section{$m R N A$ Extraction and quantitative polymerase chain reaction}

Total RNA was extracted using TRI reagent (T9424, Sigma) according to the manufacturer's instructions. Briefly, $800 \mu \mathrm{L}$ of TRI reagent was added to each sample and stored at $-80{ }^{\circ} \mathrm{C}$. After defrosting, samples were incubated with $160 \mu \mathrm{L}$ of chloroform (Sigma) for $15 \mathrm{~min}$ and $12,000 \mathrm{~g}$ centrifuged for $15 \mathrm{~min}$ at $4{ }^{\circ} \mathrm{C}$. The aqueous fraction was collected and an equal part of isopropanol (Sigma Aldrich) was added. After $10 \mathrm{~min}$, samples were centrifuged at $12,000 \mathrm{~g}$ for $10 \mathrm{~min}$ at $4{ }^{\circ} \mathrm{C}$. RNA pellet was washed with $800 \mu \mathrm{L}$ of $70 \%$ ethanol and subsequently centrifuged at 7,500 g for $5 \mathrm{~min}$ at $4{ }^{\circ} \mathrm{C}$. Air-dried RNA samples were resuspended in $15 \mu \mathrm{L}$ of RNase/DNase free water (Gibco). RNA quantity and purity were determined with a NanoDrop ND-1000 spectrophotometer (NanoDrop Technologies). The cDNA synthesis was performed with the qScript cDNA Synthesis kit (Quanta Biosciences) and using the Mastercycler Realplex (Eppendorf) using an initial amount of total RNA of $1 \mu \mathrm{g}$ in a total volume of $20 \mu \mathrm{L}$. The quantification of the transcripts was carried out by quantitative polymerase chain reaction (qPCR) using the PerfeCTA SYBR Green FastMix kit (Quanta Biosciences) following the manufacturer's protocol, in a Real-Time Mastercycler Realplex thermocycler (Eppendorf). The primers were pre-designed with PerlPrimer v1.1.21 software (Table 1) and synthesized by MWG Biotech. GAPDH (glyceraldehyde-3-phosphate dehydrogenase) was used as the housekeeping gene. The $2^{-\Delta \Delta \mathrm{Ct}}$ method was selected to evaluate the relative expression level for each target gene. ${ }^{26}$ All values were first normalized against GAPDH values, and then to the hASCs cells collected at day 0 . 
A

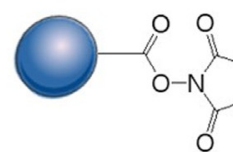

MNPs

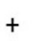

Anti-Activin

Receptor Type IIA

antibody
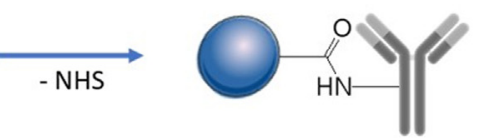

MNPs-ActRIIA

B

\begin{tabular}{lcc}
\hline Particles & Size $(\mathrm{nm})$ & Zeta potential $(\mathrm{mV})$ \\
\hline MNPs-ActRIIA & $349.43 \pm 2.08$ & $-7.1 \pm 0.6$ \\
\hline MNPs-IgG & $369.70 \pm 2.15$ & $-16.1 \pm 0.4$ \\
\hline
\end{tabular}

Figure 3. Development and characterization of functionalized MNPs complexes. A) Schematic representation of MNPs functionalization with anti-activin receptor type IIA antibody by carbodiimide activation; B) Size and zeta potential analysis of MNPs functionalized with anti-activin receptor type IIA antibody (MNPs-ActRIIA) or with IgG secondary antibody (MNPs-IgG).

\section{Quantification of collagen and non-collagenous proteins}

The amount of collagen and non-collagenous proteins expressed by hASCs cultured in 2D was determined using a semi-quantitative assay, namely Sirius Red/Fast Green Collagen Staining Kit (9046, Chondrex). After cells fixation, the dye solution was added and incubated for $30 \mathrm{~min}$ in order to completely immerse the samples. A dye extraction buffer was then mixed and the OD values read in a spectrophotometer (Synergy HT, Biotek Instruments) at $540 \mathrm{~nm}$ and $605 \mathrm{~nm}$.

\section{Immunofluorescence}

Cells were rinsed in PBS and fixed in formalin (INOPAT) prior to the detection of Tenomodulin (sc-49324) and Fibromodulin (ab81443) deposition. After cell permeabilization with $0.025 \%$ Triton-X100 (Sigma/X100)/PBS solution, the blocking step was performed using RTU Normal Horse Serum (RTU Vectastain Kit, PK-7200, Vector). Then, cells were incubated overnight with anti-tenomodulin and anti-fibromodulin antibodies, diluted in antibody diluent with background reducing components from Dako (S3022, Dako) at $4{ }^{\circ} \mathrm{C}$. Samples were then rinsed in $0.025 \%$ Triton-X100 (Sigma/X100)/PBS solution followed by incubation for $1 \mathrm{~h}$ at room temperature with the respective Alexa fluor 488 (Alfagene) considering the host species of the primary antibodies. Samples were stained with 4,6-Diamidino-2-phenyindole, dilactate (DAPI, $5 \mu \mathrm{g} / \mu \mathrm{L}$, D9564, Sigma) for $10 \mathrm{~min}$ and with Phalloidin-Tetramethylrhodamine B isothiocyanate (P1951, Sigma) for $20 \mathrm{~min}$. Samples were observed with a Fluorescence Inverted Microscope (Axio Observer, Zeiss).

\section{Statistics}

Quantitative results are expressed as the mean \pm standard deviation. Two-way ANOVA followed by Bonferroni's Multiple Comparison test was assessed to determine whether differences between sample groups were significant, unless specified. Differences were considered significant when the $P$ value was $<0.05$.

\section{Results}

Assessment of Smad2/Smad3 phosphorylation in hASCs triggered by TGF- $\beta$-like ligands

TGF- $\beta$-like ligands, Activin A and TGF- $\beta 3$ known for their functions in tendon development, ${ }^{10,27}$ were investigated as inducers of serine/threonine receptor's activation and consequent phosphorylation of Smad2/3 (Figure 2, A). Using two different ligand doses $(10$ and $20 \mathrm{ng} / \mathrm{mL})$ and at varying temporal exposures, Smad $2 / 3$ signaling and subsequent gene expression has been assessed. Moreover, Activin A and TGF- $\beta 3$ ligand-receptor binding will serve as a positive experimental control of the activation of ActRIIA receptor mediated by MNPs-antibody complexes. We investigated the levels of pSmad2/3 following 2, 10, 30 and $60 \mathrm{~min}$ of treatment.The detection of $\mathrm{pSmad} 2 / 3$ proteins was rapid (at $10 \mathrm{~min}$ ) and more intense in Activin A supplemented medium at $20 \mathrm{ng} / \mathrm{mL}$ than in medium supplemented with TGF- $\beta 3$ ligand (Figure 2, $B$ ).

\section{Development and characterization of functionalized MNPS complexes}

After antibody functionalization by carbodiimide activation (Figure 3, $A$ ), the size and surface charge of MNPs-ActRIIA and MNPs-IgG were characterized. The particle size increased after IgG coating of MNPs in comparison to MNPs coated with anti-ActRIIA (MNPs-ActRIIA). Conversely, the surface charge increased in MNPs-ActRIIA (Figure 3, $B$ ).

In order to co-localize functionalized MNPs within hASCs, MNPs-ActRIIA or MNPs-IgG labelled cells were visualized by 

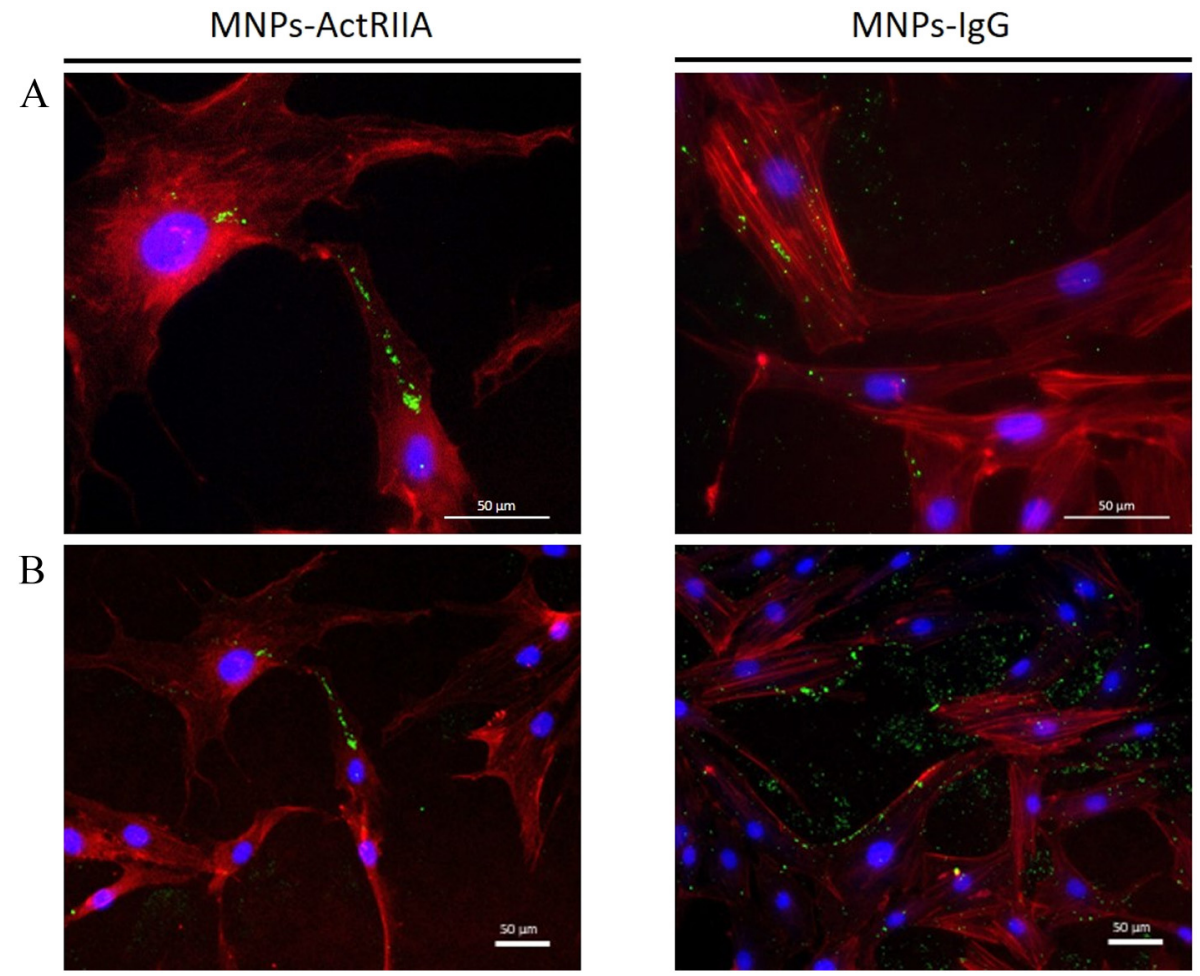

Figure 4. Dextran (green) immunofluorescence images of A) hASCs labelled with MNPs-ActRIIA or MNPs-IgG. Cell nuclei are shown by DAPI (blue) and the cytoskeleton by phalloidin (red); B) lower magnification images.

fluorescence microscopy after immuno-labelling the MNPs dextran shell with an anti-dextran antibody (Figure 4), suggesting that 30 min after labelling, there is a clear association of MNPs-ActRIIA and MNPs-IgG with cells.

Triggering Smad2/Smad3 phosphorylation in hASCs by MNPS complexes

The activin receptor activation was indirectly assessed through detection of downstream phosphorylation of Smad2/3 using an ELISA kit assay that recognizes endogenous levels of these proteins. Magneto-mechanical stimulated groups showed significantly higher phosphorylation levels in relation to non-stimulated and to Activin A control groups after 2 and 10 min, decreasing after 30 min (Figure 5, $A$ ) in MNPs-ActRIIA ST and MNPs-ActRIIA MICA conditions. The detection of phospho-Smad2/3 proteins seems to be more intense after 10min in hASCs labeled with MNPs-ActRIIA under magnetic stimulation, which highlights a more efficient ActRIIA activation. Results also show a significant elevation of absorbance values in comparison to the negative control group, MNPs-IgG, or to the positive control group, Activin (Figure 5, B).

\section{Triggering tenogenesis in hASCs using MNPs-ActRIIA complex}

In this study, real time RT-PCR analysis, the production assessment of collagen and non-collagenous proteins and the deposition of Tenomodulin (TNMD) and Fibromodulin (FMOD) tendon-related proteins were conducted up to 14 days upon
ActRIIA activation under magnetic field stimulation provided by MICA bioreactor.

\section{Gene expression analysis}

The expression of tendon associated genes was found to be higher in hASCs bound with MNPs-ActRIIA with and without the magnetic stimulation after 7 days (Figure 6). PCR results evidence higher gene expression values in MNPs-ActRIIA after 7 days under magnetic stimulation. Targeted mechano-activation of the ActRIIA using magnetic actuation of MNPs attached to the membrane receptor resulted in enhanced upregulation of tenogenic genes, TNC, TNMD, SCXA,DCN, and COL3A1 but not COL1A1. Interestingly, MNPs-ActRIIA binding alone enhanced $T N C$ expression but not other tenogenic genes, as for example $T N C$ gene expression is significantly higher in MNPs-ActRIIA ST $(P<0.05)$ in comparison to Activin A ST and to $\alpha$ MEM ST $(P<0.01)$.

\section{Extracellular matrix formation assessment}

Of the tendon dry weight, $60 \%$ is collagen type I arranged in tensile-resistant fibers ${ }^{28}$ and other types such as collagen types III ( $0-10 \%)$, IV ( $2 \%), \mathrm{V}$, and VI. The non-collagenous matrix is primarily made up of glycoproteins which include the proteoglycans such as decorin (DCN), tenascin C (TNC), fibromudulin (FMOD) and tenomodulin (TNMD). ${ }^{29} \mathrm{ECM}$ formation was assessed by production of collagen and non-collagenous proteins as depicted in Figure 7. Collagen and non-collagenous proteins 
A

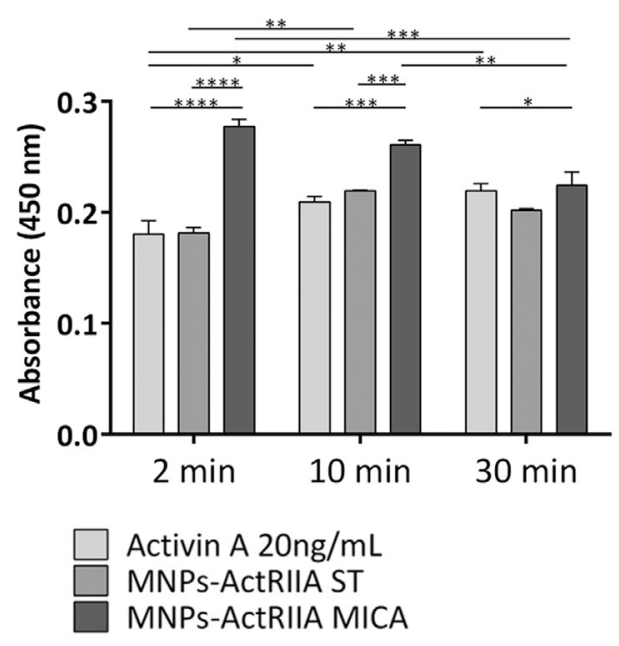

B
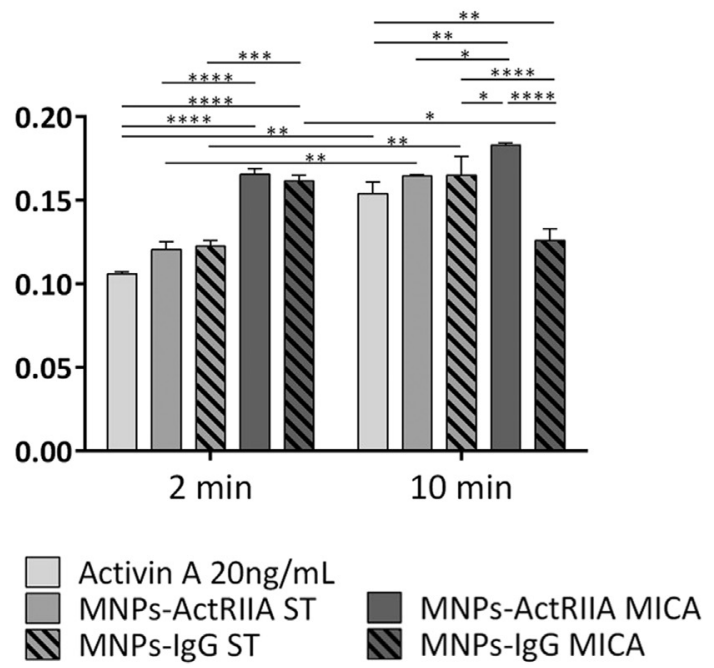

Figure 5. Phosphorylation assessment of Smad2/Smad3 in hASCs. A) Phospho-Smad2/Smad3 ELISA assay of hASCs cultured in Activin A (positive control) and with MNPs-ActRIIA under static (ST) or under actuation of a vertically oscillating magnetic field (MICA) for 2, 10, and 30min; B) Phospho-Smad2/Smad3 ELISA assay of hASCs cultured with MNPs-ActRIIA or MNPs-IgG under static (ST) or under actuation of a vertically oscillating magnetic field (MICA) for up to $10 \mathrm{~min}$; Values represent the mean $\pm \mathrm{SD}$. Symbol *,**, *** and $* * * *$ denotes study groups with statistical significant difference $P<0.05, P<0.01, P<0.001$ and $P<0.0001$ respectively.

production by hASCs significantly increased with time in culture from day 0 to 7 days and from 0 to 14 days, respectively (statistical significant groups: $a$ and $b$ ). Interestingly, the collagen values obtained in MNPs-ActRIIA MICA condition were significantly higher than in static conditions after 14 days. These values were also higher than $\alpha$ MEM or Activin A controls. The same trend was observed in non-collagenous protein production.

\section{Tendon specific markers assessment}

FMOD is highly expressed in the tendon and identified as a critical component of the tendon stem cell niche. ${ }^{30}$ The immunodetection of TNMD and FMOD is presented in Figure 8, $A$ and $B$, respectively. Tendon-specific transcription factors such as $S C X^{3}$ are essential and involved in mechanoresponsive tenogenesis through regulation of its downstream ECM proteins such as the tendon marker TNMD ${ }^{31,32}$ and proteoglycans such as FMOD. Tenomodulin and Fibromodulin proteins deposition was increased by hASCs previously labeled with MNPs-ActRIIA in comparison with hASCs cultured in basal $\alpha \mathrm{MEM}$ or in $\alpha \mathrm{MEM}$ supplemented with Activin A (negative and positive controls, respectively). Also, the detection of these proteins after 7 and 14 days of culture was higher when the oscillating magnetic field, provided by MICA bioreactor, was applied.

\section{Discussion}

Signal transduction molecules of the Smad family are components of a critical intracellular pathway that transmit signals mediated by transmembrane serine/threonine kinase type II receptors into the nucleus. Smad2/3 signaling pathways are associated with tendon development and transcriptional responses and, to date and to author's knowledge, no published studies have reported to locally target and activate Activin receptors upon mechano-magnetic stimulation, in order to modulate Smad $2 / 3$ signaling. Binding of TGF- $\beta$ family ligands, such as Activins or TGF- $\beta 3$, to receptors, leads to the recruitment, phosphorylation and activation of type I Activin receptor (ALK4), which provides a binding site for the downstream substrates, initiating the intracellular signaling through activation of Smad proteins. ${ }^{33}$ In the present work, the assessment of $\mathrm{Smad} 2 / \mathrm{Smad} 3$ phosphorylation in hASCs triggered by TGF- $\beta$-like ligands, showed increased absorbance values in Activin A supplemented medium $(20 \mathrm{ng} / \mathrm{mL})$ after 10 min, what suggests a more effective phosphorylation of Smad proteins. Therefore, Activin A at this concentration was selected as the positive control for ActRIIA activation in the subsequent experiments of this study. Indeed, the activation of the ligand-receptor complex is described as a relatively fast step in the TGF- $\beta$ signal transduction, occurring within $2 \mathrm{~min}$ after ligand stimulation, and the signal is relayed to the activation of Smad proteins, which reach at maximal levels in up to 30-60 minutes. $^{34}$

In order to remotely stimulate ActRIIA receptor in a controlled way, MNPs complexes were developed and characterized. The results of size and surface charge of the developed complexes (MNPs-ActRIIA and MNPs-IgG) suggested improved electrostatic interactions of the MNPs coated with anti-ActRIIA with the negatively charged cell membrane, which were further co-localized in hASCs after the $30 \mathrm{~min}$ period of labelling by immunocytochemistry.

To understand the capability of functionalized MNPs (MNPs-ActRIIA) to directly target and remotely activate the 
TNC

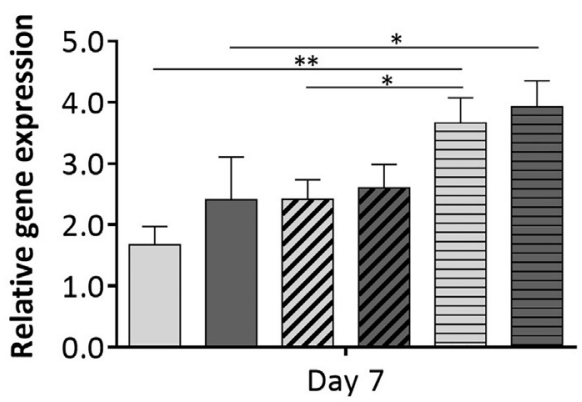

$D C N$

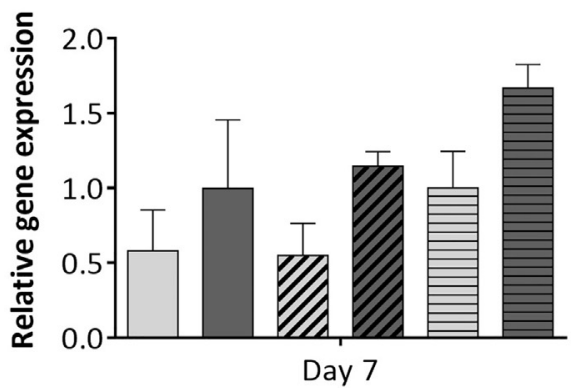

TNMD

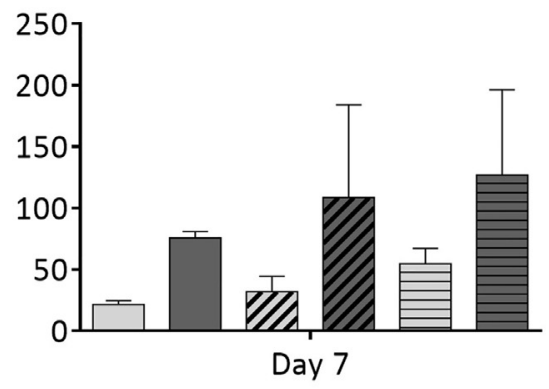

COL1A1

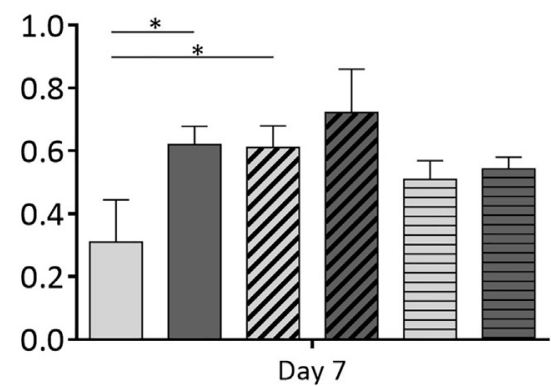

SCXA

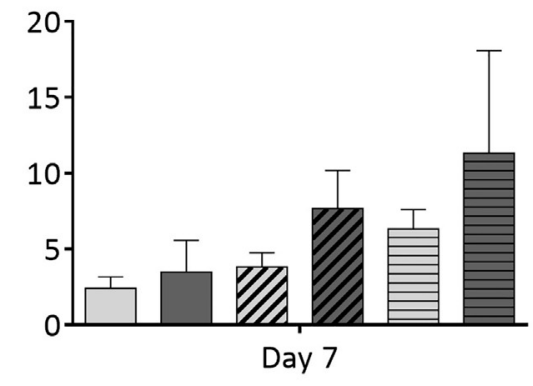

COL3A1

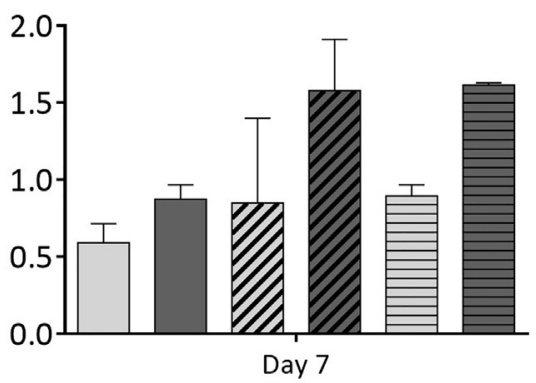

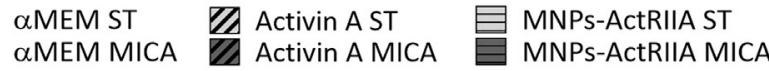

Figure 6. Real time RT-PCR analysis of tenascin C (TNC), tenomodulin (TNMD), scleraxis (SCXA), decorin (DCN), collagen type I (COL1A1) and collagen type III (COL3Al) gene expression of hASCs cultured for 7 days in $\alpha$ MEM medium, $\alpha$ MEM supplemented with Activin A (20 ng/mL) or labeled with MNPs-ActRIIA in $\alpha$ MEM medium, under static (ST) or under actuation of a vertically oscillating magnetic field (MICA). Values represent the mean \pm SD. Symbol * and ** denotes study groups with statistical significant difference $P<0.05$ and $P<0.01, P<0.001$, by one-way ANOVA.

Activin receptor via magneto-mechanical stimulation, we investigated the phosphorylation of Smad2/3 using MICA bioreactor and comparing to static environment. The outcomes suggested that ActRIIA is a mechanosensitive receptor that can be remotely activated using $\mathrm{Ab}$ coated MNPs, whose action is favored by the actuation of an external magnetic field provided by MICA bioreactor. Previous works using biofunctionalized MNPs targeted to mechano-responsive receptors demonstrated an enhanced mechanoactivation of the targeted receptors caused by movement of the MNPs-Ab complex in the magnetic field.14,21,35 Furthermore, the commitment of hASCs towards tenogenesis using the MNPs-ActRIIA complex was studied. Tenoblasts maturation to tenocytes is characterized by the formation of enriched ECM and controlled by key tenogenic transcription factors, such as $S C X .^{36}$ The TGF- $\beta$ signaling pathway regulates a diverse group of cellular behaviors such as proliferation, differentiation, and growth arrest ${ }^{37}$ and has also been suggested as the most active pathway in tendon cells during mouse limb development. ${ }^{38}$ Overall, magnetic actuation of MNPs coated with ActRIIA bound to the receptor enhanced the expression of all tendon related genes studied. Havis et al. reported that blocking the TGF- $\beta$ pathway with SB43 inhibitor, significantly downregulated SCX and COL1a1, among other gene expression ${ }^{38}$ concluding that TGF- $\beta$ is required via the Smad2/3 intracellular pathway for expression of tendon-related markers in mouse forelimbs. ${ }^{38}$ We can infer that MNPs-ActRIIA and magnetic actuation synergistically influenced Smad2/3 mediators. This agrees with other studies using alternate mechanical activation methods where phosphorylation assays, and $S C X$ and TNMD transcriptional activity was upregulated in response to strain. ${ }^{12,31,39}$ Furthermore, the fluctuations in gene expression from 7 to 14 days (Figure S1) may be related with ligand depletion from the environment causing cells to lose the stimulation and shut down the phosphorylation of Smad2, which can occur within a few hours post stimulation. ${ }^{40,41}$ Dosage and stimulation time of TGF- $\beta$-like ligands have effects on Smad signaling and short-term ligand pulse stimulation results in transient pSmad2, whereas serial pulses or continuous stimulation results in sustained pSmad2. ${ }^{40}$ Moreover, there is a time-delay for reaching the maximum $\mathrm{pSmad} 2 / 3$ phosphorylation, which we determined to be $10 \mathrm{~min}$ after treatment with MNPs-ActRIIA or with Activin A supplementation at $20 \mathrm{ng} / \mathrm{mL}$, and nuclear accumulation and subsequent regulation of transcriptional responses. Considering these two assertions and the results of gene expression we can infer that as the complex MNPs-ActRIIA was provided to hASCs once at the beggining of 
Collagen

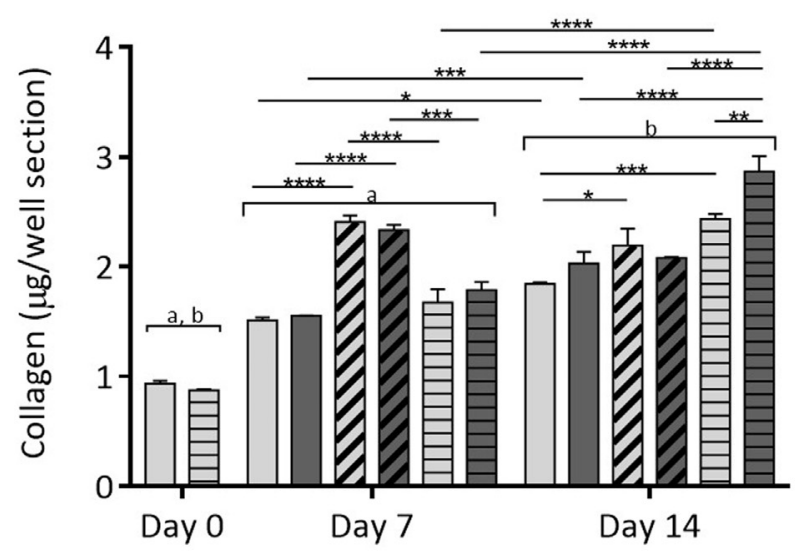

Non-collagen

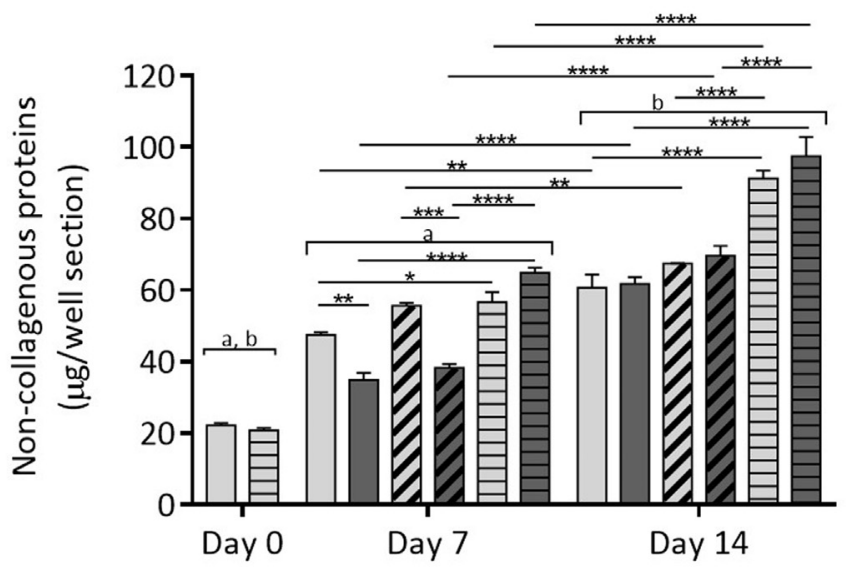

QMEM ST aMEM MICA

Activin A ST
Activin A MICA

MNPs-ActRIIA ST

MNPs-ActRIIA MICA

Figure 7. Quantification of collagen and non-collagenous proteins by Sirius Red/Fast Green Collagen staining kit in hASCs labeled with MNPs-ActRIIA in $\alpha$ MEM medium, under static (ST) or under actuation of a vertically oscillating magnetic field (MICA). hASCs cultured for up to 14 days in $\alpha$ MEM medium and in $\alpha$ MEM supplemented with Activin A $(20 \mathrm{ng} / \mathrm{mL})$ were considered the negative and positive experimental controls, respectively. Values represent the mean \pm $\mathrm{SD}$. Symbol *, **,*** and **** denotes study groups with statistical significant difference $P<0.05, P<0.01, P<0.001$ and $P<0.0001$ respectively; a and $\mathrm{b}$ letters represent statistically different groups.

culture, the transcriptional responses observed were time-dependent and transient mostly affecting gene expression in early culture periods.

Additionally, the synthesis of a complex matrix of collagen and non-collagenous proteins also supports the activation of activin receptor by MNPs-ActRIIA complex under magnetic actuation in a more efficient way than using the Activin A ligand to trigger hASCs response. Considering the role of TNMD as specific tendon marker and FMOD as a crucial proteoglycan component of tendons that regulate physiological ECM assembly, it was assessed the deposition of these markers by hASCs tagged MNPs-ActRIIA. Tenomodulin has been sugested to be expressed upon Activin II receptor stimulation by myostatin, triggering the $\mathrm{Smad} 2 / 3$ signaling cascade and increasing the expression of $S C X$ that ultimately results in the TNMD gene expression. ${ }^{10}$ Thus, upon MICA stimulation, MNPs-ActRIIA complexes are likely to act over Activin II receptor, activating the signaling cascade and ultimately inducing the transcription of TNMD and its translation into protein. Moreover, FMOD was suggested to play a role in the formation of ECM and to regulate TGF- $\beta$ levels. ${ }^{42}$ Also, mutations in the small leucine-rich proteoglycan gene FMOD result in irregularities in the diameter of collagen fibers in the tendons. ${ }^{43}$ Herein, the higher deposition of TNMD and FMOD together with Sirius Red/Fast Green Collagen staining assay and RT-PCR, confirms the hypothesis of magnetically actuated MNPs-tagged hASCs towards a tenogenic commitment. Moreover, it was clear in the present work that the induction of Smad2/3 signaling elicited hASCs tenogenic response. However, it is known that other variables outside the target cell determine the extent of stimulation by TGF- $\beta$ cytokines. ${ }^{44}$ Thus, future studies will take into account the impact of extracellular regulators in Smad signaling dynamics, such as ligand exposure, or the effect of antagonistic ligands.

Collectively, the present work has shown that the remote activation of MNPs tagging mechanosensitive receptors of hASCs may have potential for controlling stem cell differentiation envisioning successful cell therapies for tendon regeneration. Previously, we have shown the potential benefit of this apporach in orthopaedic bone tissue engineering and similar strategies could be employed for tendon regeneration. ${ }^{13}$ It has been demonstrated that magnetic MNPs tagging can be utilised in tendon lineage comitment via ActRIIA/Smad2/3 signaling cascade, as shown by phosphorylation of $\mathrm{Smad} 2 / 3$ proteins in MNPs-ActRIIA tagged hASCs. Further investigation is required to fully explore the mechanism behind signal activation and downstream signaling events in response to MNPs-ActRIIA stimulation and the effects on hASCs fate and differentiation. The development of this technology raises the possibility of remotely controlling TGF- $\beta / \mathrm{Smad} 2 / 3$ signaling and consequently the control of stem cell behavior. Overall, the remote activation of MNPs tagged hASCs may have potential for controlling stem cell differentiation following cell therapy and modulation of signaling pathways involved in tendon regeneration.

\section{Appendix A. Supplementary data}

Supplementary data to this article can be found online at https://doi.org/10.1016/j.nano.2018.02.008. 
A

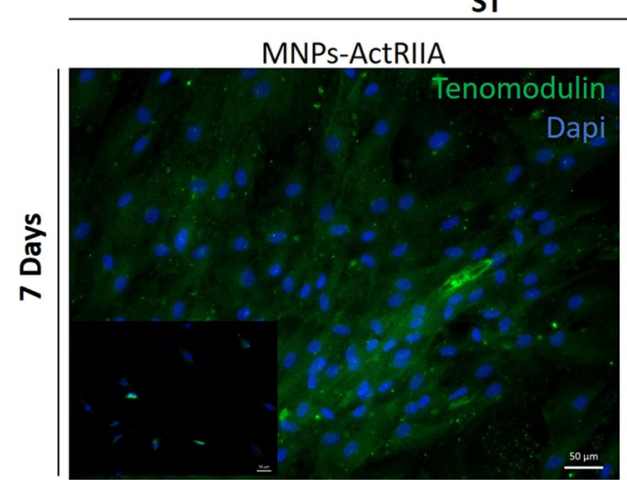

MNPs-ActRIIA

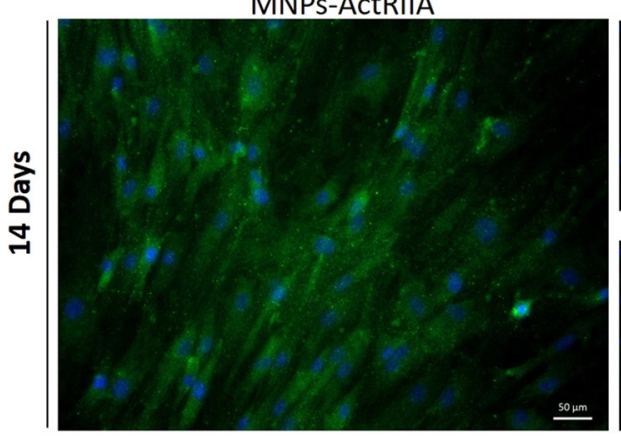

B

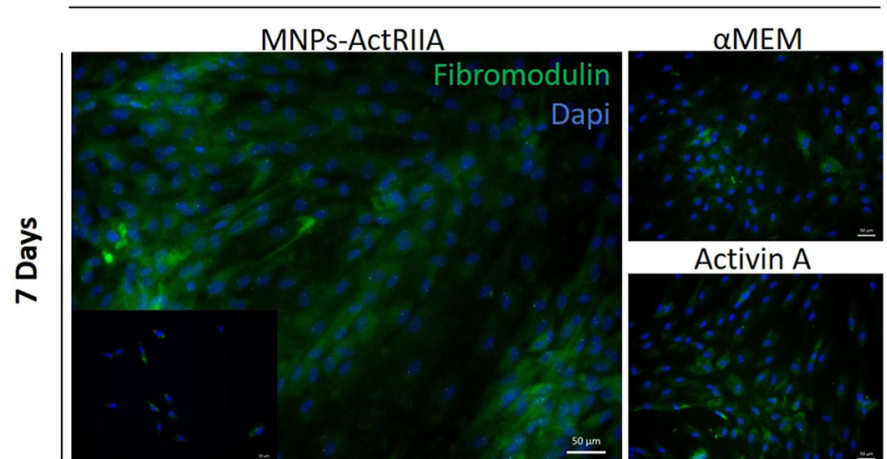

MNPs-ActRIIA

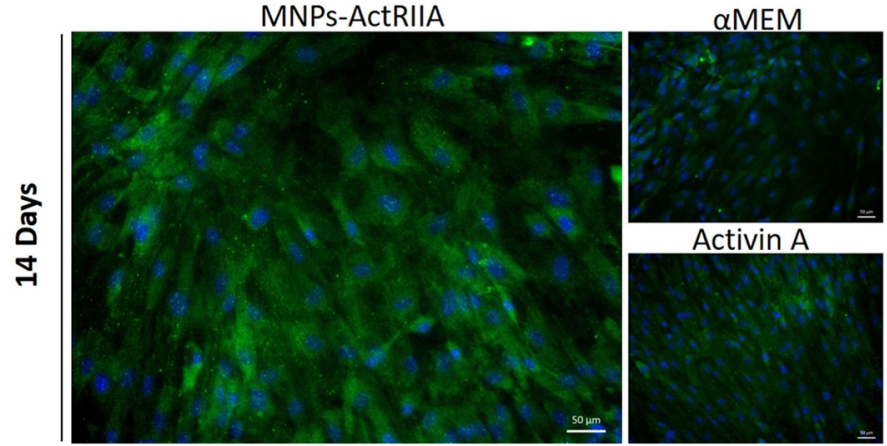

\section{MICA}

MNPs-ActRIIA

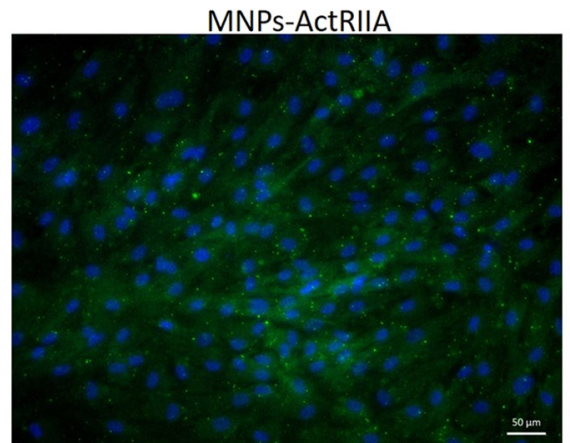

MNPs-ActRIIA
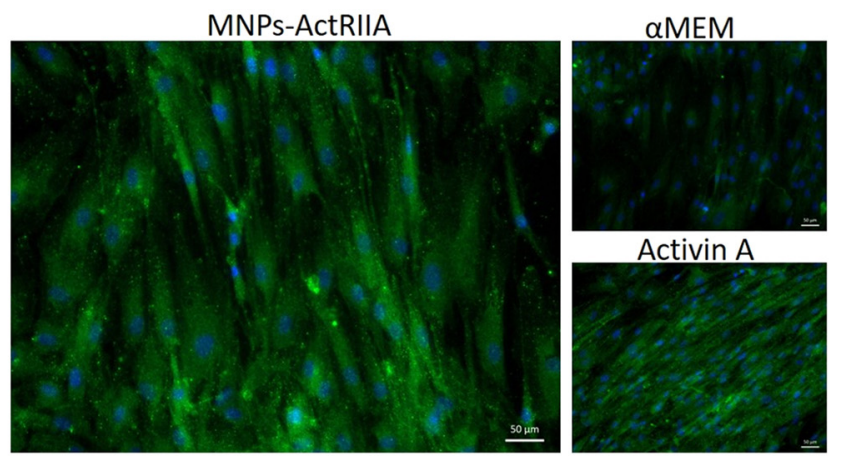

\section{MICA}

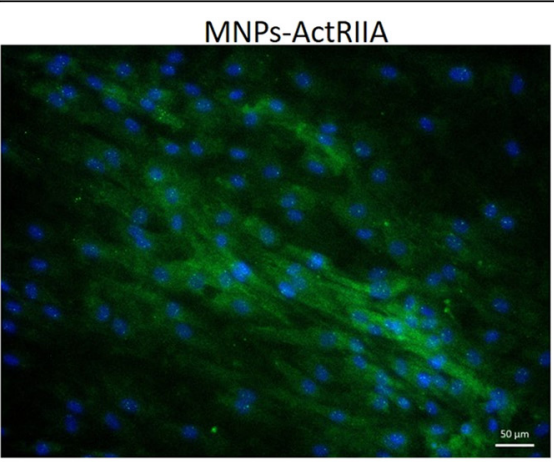

MNPs-ActRIIA

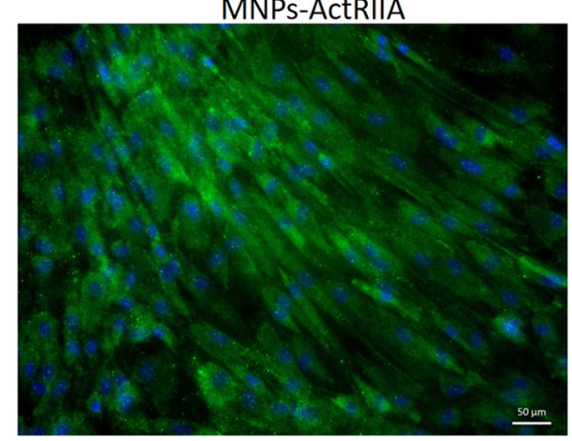

Activin A

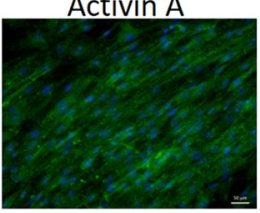

aMEM

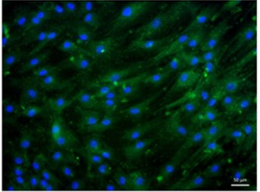

Activin A

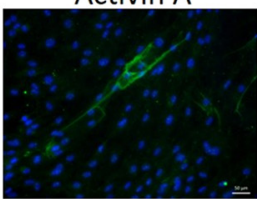

QMEM

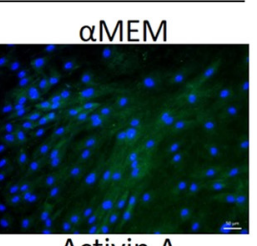

Activin A

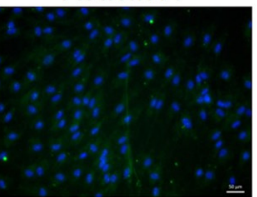

$\alpha \mathrm{MEM}$

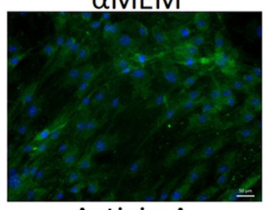

Activin A

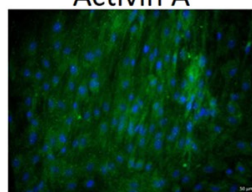

Figure 8. Immunocytochemistry of A) Tenomodulin and of B) Fibromodulin proteins (green) deposited by hASCs labeled with MNPs-ActRIIA in $\alpha$ MEM medium, under static (ST) or under actuation of a vertically oscillating magnetic field (MICA). hASCs cultured for up to 14 days in $\alpha$ MEM medium and in $\alpha$ MEM supplemented with Activin A $(20 \mathrm{ng} / \mathrm{mL})$ were considered the negative and positive experimental controls, respectively. DAPI (blue) stains cell nuclei. Insets represent hASCs labeled with MNPs-ActRIIA in $\alpha$ MEM medium at day 0 . 


\section{References}

1. Wall ME, Dyment NA, Bodle J, Volmer J, Loboa E, Cederlund A, et al. Cell Signaling in Tenocytes: Response to Load and Ligands in Health and Disease. Adv Exp Med Biol 2016;920:79-95.

2. Wall M, Butler D, Haj AE, Bodle JC, Loboa EG, Banes AJ. Key developments that impacted the field of mechanobiology and mechanotransduction. J Orthop Res 2017;36(92):,605-619.

3. Gaut L, Duprez D. Tendon development and diseases. Wiley Interdiscip Rev Dev Biol 2016;5(1):5-23.

4. Havis E, Bonnin MA, Esteves de Lima J, Charvet B, Milet C, Duprez D. TGFbeta and FGF promote tendon progenitor fate and act downstream of muscle contraction to regulate tendon differentiation during chick limb development. Development 2016;143(20):3839-51.

5. Wakefield LM, Hill CS. Beyond TGF $\beta$ : roles of other TGF $\beta$ superfamily members in cancer. Nat Rev Cancer 2013;13(5):328-41.

6. Rothrauff BB, Yang G, Tuan RS. Tendon resident cells-functions and features in section I-developmental biology and physiology of tendons. Tendon regeneration. Elsevier Inc.; 2015. p. 41-76.

7. Jones ER, Jones GC, Legerlotz K, Riley GP. Cyclical strain modulates metalloprotease and matrix gene expression in human tenocytes via activation of TGFß. Biochim Biophys Acta 1833;2013:2596-607.

8. Pauklin S, Vallier L. Activin/Nodal signalling in stem cells. Development 2015;142(4):607-19.

9. Yu L, Hebert MC, Zhang YE. TGF-beta receptor-activated p38 MAP kinase mediates Smad-independent TGF-beta responses. EMBO J 2002;21(14):3749-59.

10. Qi J, Dmochowski JM, Banes AN, Tsuzaki M, Bynum D, Patterson M, et al. Differential expression and cellular localization of novel isoforms of the tendon biomarker tenomodulin. J Appl Physiol 2012;113 (6):861-71.

11. Brandau O, Meindl A, Fassler R, Aszodi A. A novel gene, tendin, is strongly expressed in tendons and ligaments and shows high homology with chondromodulin-I. Dev Dyn 2001;221(1):72-80.

12. Docheva D, Hunziker EB, Fässler R, Brandau O. Tenomodulin is necessary for tenocyte proliferation and tendon maturation. Mol Cell Biol 2005;25(2):699-705.

13. Henstock JR, Rotherham M, Rashidi H, Shakesheff KM, El Haj AJ. Remotely activated mechanotransduction via magnetic nanoparticles promotes mineralization synergistically with bone morphogenetic protein 2: applications for injectable cell therapy. Stem Cells Transl Med 2014;3(11):1363-74.

14. Rotherham M, El Haj AJ. Remote activation of the Wnt/beta-catenin signalling pathway using functionalised magnetic particles. PLoS One 2015;10(3)e0121761.

15. Gomes ME, Rodrigues MT, Domingues RMA, Reis RL. Tissue engineering and regenerative medicine: new trends and directions - a year in review. Rev 2017;23(3):211-24.

16. Maziarz A, Kocan B, Bester M, Budzik S, Cholewa M, Ochiya T, et al. How electromagnetic fields can influence adult stem cells: positive and negative impacts. Stem Cell Res Ther 2016;7(1):54-65.

17. de Girolamo L, Vigano M, Galliera E, Stanco D, Setti S, Marazzi MG, et al. In vitro functional response of human tendon cells to different dosages of low-frequency pulsed electromagnetic field. Knee Surg Sports Traumatol Arthrosc 2015;23(11):3443-53.

18. Xu D, Zhang T, Qu J, Hu J, Lu H. Enhanced Patella-Patellar Tendon Healing Using Combined Magnetic Fields in a Rabbit Model. Sports Med 2014;42(10):2495-501.

19. Strauch B, Patel MK, Rosen DJ, Mahadevia S, Brindzei N, Pilla AA. Pulsed magnetic field therapy increases tensile strength in a rat Achilles' tendon repair model. J Hand Surg Am 2006;31(7):1131-5.

20. Kanczler JM, Sura HS, Magnay J, Green D, Oreffo RO, Dobson JP, et al. Controlled differentiation of human bone marrow stromal cells using magnetic nanoparticle technology. Tissue Eng A 2010;16(10):3241-50.
21. Hu B, Dobson J, Haj AJE. Control of smooth muscle $\alpha$-actin (SMA) upregulation in HBMSCs using remote magnetic particle mechanoactivation. Nanomedicine 2014;10:45-55.

22. Russell JW, Russell TM, Vasey JR, Hall MS. Autologous bone marrow aspirate for treatment of superficial digital flexor tendonitis in 105 racehorses. Vet $\operatorname{Rec}$ 2016;179(3):69.

23. Rada T, Reis RL, Gomes ME. Novel method for the isolation of adipose stem cells (ASCs). J Tissue Eng Regen Med 2009;3(2):158-9.

24. Carvalho PP, Wu X, Yu G, Dias IR, Gomes ME, Reis RL, et al. The effect of storage time on adipose-derived stem cell recovery from human lipoaspirates. Cells Tissues Organs 2011;194(6):494-500.

25. Attisano L, Wrana JL, Montalvo E, Massague J. Activation of signalling by the activin receptor complex. Mol Cell Biol 1996;16(3):1066-73.

26. Livak KJ, Schmittgen TD. Analysis of relative gene expression data using real-time quantitative PCR and the $2-\Delta \Delta \mathrm{CT}$ method. Methods 2001;25(4):402-8.

27. Pryce BA, Watson SS, Murchison ND, Staverosky JA, Dunker N, Schweitzer R. Recruitment and maintenance of tendon progenitors by TGFbeta signaling are essential for tendon formation. Development 2009;136(8):1351-61.

28. Kjaer M. Role of extracellular matrix in adaptation of tendon and skeletal muscle to mechanical loading. Physiol Rev 2004;84(2):649-98.

29. Thorpe CT, Birch HL, Clegg PD, Screen HR. The role of the noncollagenous matrix in tendon function. Exp Pathol 2013;94(4):248-59.

30. Bi Y, Ehirchiou D, Kilts TM, Inkson CA, Embree MC, Sonoyama W, et al. Identification of tendon stem/progenitor cells and the role of the extracellular matrix in their niche. Nat Med 2007;13(10):1219-27.

31. Shukunami C, Takimoto A, Oro M, Hiraki Y. Scleraxis positively regulates the expression of tenomodulin, a differentiation marker of tenocytes. Dev Biol 2006;298(1):234-47.

32. Murchison ND, Price BA, Conner DA, Keene DR, Olson EN, Tabin CJ, et al. Regulation of tendon differentiation by scleraxis distinguishes force-transmitting tendons from muscle-anchoring tendons. Development 2007;134(14):2697-708.

33. Abe Y, Minegishi T, Leung PC. Activin receptor signaling. Growth Factors 2004;22(2):105-10.

34. Zi Z, Chapnick DA, Liu X. Dynamics of TGF-beta/Smad signaling. FEBS Lett 2012;586(14):1921-8.

35. Rotherham M, Henstock JR, Qutachi O, El Haj AJ. Remote regulation of magnetic particle targeted Wnt signaling for bone tissue engineering. Nanomedicine 2017;14(1):173-84.

36. Spanoudes K, Gaspar D, Pandit A, Zeugolis DI. The biophysical, biochemical, and biological toolbox for tenogenic phenotype maintenance in vitro. Trends Biotechnol 2014;32(9):474-82.

37. Massagué J. TGF- $\beta$ signal transduction. Annu Rev Biochem 1998;67(1):753-91.

38. Havis E, Bonnin M-A, Olivera-Martinez I, Nazaret N, Ruggiu M, Weibel J, et al. Transcriptomic analysis of mouse limb tendon cells during development. Development 2014;141(19):3683-96.

39. Maeda T, Sakabe T, Sunaga A, Sakai K, Rivera AL, Keene DR, et al. Conversion of mechanical force into TGF- $\beta$ mediated biochemical signals. Curr Biol 2011;21(11):933-41.

40. Zi Z, Feng Z, Chapnick DA, Dahl M, Deng D, Klipp E, et al. Quantitative analysis of transient and sustained transforming growth factor-beta signaling dynamics. Mol Syst Biol 2011;7:492-503.

41. Feng Z, Zi Z, Liu X. Measuring TGF-beta ligand dynamics in culture medium. Methods Mol Biol 2016;1344:379-89.

42. Walden G, Liao X, Donell S, Raxworthy MJ, Riley GP, Saeed A. A clinical, biological, and biomaterials perspective into tendon injuries and regeneration. Rev 2017;23(1):44-58.

43. Svensson L, Aszodi A, Reinholt FP, Fassler R, Heinegard D, Oldberg A. Fibromodulin-null mice have abnormal collagen fibrils, tissue organization, and altered lumican deposition in tendon. J Biol Chem 1999;274 (14):9636-47.

44. Massagué J. TGF $\beta$ signalling in context. Nat Rev Mol Cell Biol 2012;13 (10):616-30. 